\title{
TOC e ABM: Uma Análise Comparativa à Luz da Literatura Especializada em Sistemas de Informações Gerenciais
}

Carlos Roberto Kerber

Mestrando em Ciências Contábeis pela Universidade Federal do Rio de Janeiro - UFRJ Av. Pasteur, 250 - Sala 242. Praia Vermelha. Rio de Janeiro/RJ. CEP: 22290-240

E-mail: crkerber@hotmail.com

Rodrigo Barreiros Leal Mestrando em Ciências Contábeis da Universidade Federal do Rio de Janeiro - UFRJ Av. Pasteur, 250 - Sala 242. Praia Vermelha. Rio de Janeiro/RJ. CEP: 22290-240 E-mail: rodrigobl77@hotmail.com

Ronaldo Francisco dos Santos Mestrando em Ciências Contábeis da Universidade Federal do Rio de Janeiro - UFRJ Av. Pasteur, 250 - Sala 242 - Praia Vermelha. Rio de Janeiro/RJ. CEP: 22290-240 E-mail: ronaldo.santos@kntsbrasil.com.br

Anderson Chaves Da Silva Mestrando em Ciências Contábeis da Universidade Federal do Rio de Janeiro - UFRJ Av. Pasteur, 250 - Sala 242. Praia Vermelha. Rio de Janeiro/RJ. CEP: 22290-240 E-mail: azelha@globo.com

Carlos Alexandre Nascimento Wanderley Mestrando em Ciências Contábeis da Universidade Federal do Rio de Janeiro - UFRJ Av. Pasteur, 250 - Sala 242. Praia Vermelha. Rio de Janeiro/RJ. CEP: 22290-240 E-mail: chapuwand@hotmail.com

Samuel Cogan Doutorado em Engenharia de Produção pela Universidade Federal de Rio de Janeiro UFRJ Professor da Universidade Federal do Rio de Janeiro - UFRJ Av. Pasteur, 250 - Sala 242 - Praia Vermelha. Rio de Janeiro/RJ. CEP: 22290-240 E-mail: scogan@facc.ufrj.br

\section{RESUMO}

Este artigo tem por objetivo promover uma análise comparativa entre dois sistemas de informações contábeis (SIC), à luz da literatura especializada: a Teoria das Restrições (Theory of Constraints- TOC); e a Gestão Baseada em Atividades (Activity Based Management - ABM); sob os aspectos de três dimensões para verificar se exercem a função de um sistema de informação propriamente dita. Motivado pelo surgimento de diversos modelos de SIC que prometem agregar qualidade a informação útil à tomada de decisão, procurou-se neste trabalho evidenciar os aspectos e características 
inerentes de cada um. Os aspectos metodológicos compreendem uma revisão bibliográfica que procura explicar e discutir o tema proposto, aliada a uma análise comparativa. Estruturalmente, é realizada uma revisão de literatura acerca de Sistema de Informação (SI), Teoria das Restrições (TOC) e Gestão Baseada em Atividades (ABM). O procedimento de comparação é parametrizado por três dimensões (finalidade, eficácia e sucesso) visando observar pontos como abrangência e alcance. Nesse ínterim, a pesquisa procura identificar os pontos semelhantes, bem como as relevantes diferenças entre os referidos SIC. Em linhas gerais, foi possível concluir algumas semelhanças e diferenças entre a TOC e a ABM e que, mesmo com algumas restrições ou o não atendimento de algum requisito, atendem a finalidade a que se propõem de forma eficaz e com o sucesso esperado.

Palavras-Chave: Sistema de Informação. Teoria das Restrições. Gestão Baseada em Atividades.

\section{TOC and ABM: A Comparative Analysis in the Light of Literature Specializing in Management Information Systems}

\section{ABSTRACT}

This article aims to provide a comparative analysis between two systems of accounting information $(\mathrm{SCl})$ in the light of literature: the Theory of Constraints (Theory of Constraints, TOC) and Activity Based Management (Activity Based Management - ABM ) under the aspects of three dimensions to determine whether performing the function of an information system itself. Motivated by the emergence of several models of $\mathrm{SCl}$ that promise to add quality useful information for decision making, it was evidence in this paper the aspects and characteristics of each one. Methodological issues include a literature review that seeks to explain and discuss the proposed topic, coupled with a comparative analysis. Structurally, a review of literature on information system (IS), Theory of Constraints (TOC) and Activity Based Management (ABM). The comparison procedure is parameterized by three dimensions (purpose, effectiveness and success), aiming to check items such as scope and reach. Meanwhile, the research seeks to identify the points of similarity, as well as relevant differences between those SIC. In general, it was possible to conclude some similarities and differences between TOC and $\mathrm{ABM}$, and that even with some restrictions or nonfulfillment of some requirement, serve the purpose for which they propose to effectively and with the expected success.

Keywords: Information System, Theory of Constraints; Activity Based Management.

\section{CONSIDERAÇÕES INICIAIS}

Atualmente o que se verifica, muito em função da concorrência estabelecida nos mercados que obrigam as empresas a gerirem seus negócios com grande eficiência e 
TOC e ABM: Uma Análise Comparativa à Luz da Literatura Especializada em Sistemas de

Carlos Roberto Kerber, Rodrigo Barreiros Leal, Ronaldo Francisco dos Santos, Anderson Chaves Da Silva, Carlos Alexandre Nascimento Wanderley, Samuel Cogan

velocidade, é a busca por modelos de controles que abarcam o máximo de informações de qualidade e pertinentes a tomada de decisão de seus administradores. Nesse contexto, as empresas e organizações buscam garantir suas permanências no mercado, experimentando novas formas de administrar e controlar seus patrimônios e processos de produção.

Via de regra, a vantagem competitiva é evidenciada nas organizações onde estão presentes informações de qualidade superior produzidas, na maioria das vezes, por sistemas de informações (SI) eficientes. De fato, a contabilidade exerce papel de destaque no auxílio aos gestores em meio ao processo de tomada de decisão e, para potencializar essa sua importante função, utiliza-se de sistemas de informações contábeis (SIC) de modo a organizar todo o seu arcabouço informativo.

Motivado pelo surgimento de diversos modelos de SIC que prometem agregar qualidade a informação útil à tomada de decisão, foi desenvolvido este artigo que tem por objetivo promover uma análise comparativa, à luz da literatura especializada, entre dois sistemas de informações contábeis: a Teoria das Restrições (Theory of Constraints- TOC), desenvolvida por Goldratt, cujos principais conceitos estão definidos em sua obra "A Meta", publicada em 1984; e a Gestão Baseada em Atividades (Activity Based Management - ABM), desenvolvida nos anos 1990 nos EUA, divulgada por Kaplan e Cooper.

A metodologia utilizada no trabalho consiste em uma revisão bibliográfica apoiada em consulta a livros, teses, dissertações, revistas e sítios da Internet que tratam de assuntos pertinentes ao objetivo da pesquisa. Estruturalmente, é realizada uma revisão de literatura acerca de Sistema de Informação (SI), Teoria das Restrições (TOC) e Gestão Baseada em Atividades (ABM). Em seguida, é procedida uma análise comparativa entre os SIC, parametrizada por três dimensões (finalidade, eficácia e sucesso), para verificar pontos como abrangência e alcance. Por fim, são registradas as considerações finais. 
TOC e ABM: Uma Análise Comparativa à Luz da Literatura Especializada em Sistemas de

Informações Gerenciais

Carlos Roberto Kerber, Rodrigo Barreiros Leal, Ronaldo Francisco dos Santos, Anderson Chaves Da Silva, Carlos Alexandre Nascimento Wanderley, Samuel Cogan

\section{REFERENCIAL TEÓRICO}

\subsection{Sistemas de Informação}

São diversas as definições e conceitos encontrados na doutrina acerca dos Sistemas de Informação (SI). Segundo Strassburg et al. (2007), os SI são utilizados pelas empresas principalmente para agilizar o processo de tomada de decisão, disponibilizando informações oportunas e em tempo real aos tomadores de decisão.

De acordo com Padoveze (2007) e Dias et al. (2003), o SI pode ser conceituado como um conjunto de recursos humanos, materiais, tecnológicos e financeiros que são agregados de acordo com uma sequência lógica visando o processamento dos dados e sua tradução em informações para, mediante seu produto, propiciar às organizações condições de cumprir seus objetivos principais.

Riella et al. (2003) assinalam que as empresas que possuem um Sistema de Informação apresentam um diferencial que garante a redução de custos, maior agilidade nos processos e agregam valor às atividades empresariais. Estes sistemas são aperfeiçoados com o objetivo de fornecer informações mais completas, de maneira mais rápida e com maior precisão aos usuários. Os processos são otimizados, visando prover maior ganho para a empresa.

Para Laudon e Laudon (1999), sistema de informações é um conjunto de componentes inter-relacionados que trabalham para coletar, processar, armazenar e distribuir a informação, visando facilitar o planejamento, o controle, a coordenação, a análise e o processo decisório em empresas e outras organizações. Os SI contêm informações sobre pessoas, lugares e coisas de interesse, no ambiente externo e interno da organização. Eles essencialmente transformam a informação em uma forma utilizável para a coordenação de fluxo de trabalho de uma empresa, ajudando empregados ou gerentes a tomar decisões, analisar e visualizar assuntos complexos e resolver outros tipos de problemas.

Segundo Oliveira (1999), um sistema apresenta em seu funcionamento os seguintes componentes: objetivos; entradas; processo de transformação; saídas; controle e avaliação do sistema; e retroalimentação ou realimentação. Por sua vez, Alter (1996) identifica seis elementos necessários à compreensão do papel de um SI 
TOC e ABM: Uma Análise Comparativa à Luz da Literatura Especializada em Sistemas de

em um processo de negócio: clientes; produtos; processo de negócio; participantes; informação; e tecnologia.

O'Brien (2004) evidencia que os sistemas de informação desempenham três papéis dentro de uma organização: suporte de seus processos e operações, suporte na tomada de decisão de seus funcionários e gerentes, suporte em suas estratégias em busca da vantagem competitiva.

Pelo fato de existirem diferentes interesses e níveis hierárquicos numa organização, também existem diferentes tipos de SI, segundo os tipos de decisões a serem tomadas. Uma das recomendações mais adotadas para a classificação de sistemas é a que vai ao encontro da teoria dos níveis de gestão, como citam Meirelles (1994) e Rocha (2001).

Assim, os sistemas podem ser classificados como:

- Sistemas de nível operacional: visam apoiar os processos de decisões do tipo como um dos elementos críticos para determinar o sucesso do investimento.

\subsubsection{Componentes para avaliação da dimensão de finalidade de um SI}

Segundo Oliveira (1999), as principais finalidades de um SI são:

- Reduzir custos: visa diminuir os custos das operações, da mão-de-obra burocrática e do grau de centralização de decisões na empresa;

- Aperfeiçoamento das informações: tem como propósito melhorar o acesso às informações, propiciando relatórios mais precisos e rápidos, com menor preço;

- Ganhar produtividade: objetiva melhorar a produtividade, tanto setorial quanto global, e os serviços realizados e oferecidos;

- Melhorar a tomada de decisões: visa aprimorar os processos de tomadas de decisões, por meio do funcionamento de informações mais rápidas e precisas, e estimular maior interação entre os tomadores de decisão;

- Projeção de resultados: fornecer melhores projeções dos efeitos das decisões;

- Aperfeiçoamento organizacional: melhorar a estrutura organizacional, por facilitar o fluxo de informações;

- Estruturação de poder: melhorar a estrutura de poder para aqueles que 
TOC e ABM: Uma Análise Comparativa à Luz da Literatura Especializada em Sistemas de

Carlos Roberto Kerber, Rodrigo Barreiros Leal, Ronaldo Francisco dos Santos, Anderson Chaves Da Silva, Carlos Alexandre Nascimento Wanderley, Samuel Cogan

entendem e controlam o sistema;

- Capacidade de resposta aos acontecimentos: melhorar a adaptação da empresa para enfrentar os acontecimentos não-previstos, a partir das constantes mutações nos fatores ambientais;

- Desenvolver relacionamentos: melhorar a interação com os fornecedores, assim como nas atitudes e atividades dos funcionários da empresa;

- Motivar pessoal: aumentar o nível de motivação das pessoas envolvidas; e

- Distribuir hierarquia: reduzir os níveis hierárquicos.

\subsubsection{Componentes para avaliação da dimensão de eficácia para um SI}

Polloni (2000) afirma que um sistema de informação deve atingir o mais rapidamente possível seus objetivos de armazenamento e fornecimento de informações para a organização, em formato, tempo e custos apropriados. Assim, um sistema de informação eficaz deve:

- Produzir informações: produzir informações realmente necessárias, confiáveis, em tempo hábil e com custo condizente, atendendo aos requisitos operacionais e gerenciais da tomada de decisão;

- Regular: ter por base de diretrizes capazes de assegurar a realização dos objetivos, de maneira direta, simples e eficiente;

- Integrar: integrar-se à estrutura da organização e auxiliar na coordenação das diferentes unidades organizacionais;

- Ter fluxo de processos: ter um fluxo de procedimento (interno e externo ao processamento) racional, integrado, rápido e de menor custo possível;

- Ter confiabilidade: contar com dispositivos internos que garantam a confiabilidade das informações de saída e adequada proteção aos dados controlados pelo sistema; finalmente, ser simples, seguro e rápido em sua operação. 
TOC e ABM: Uma Análise Comparativa à Luz da Literatura Especializada em Sistemas de

\subsubsection{Componentes para avaliação da dimensão de sucesso de um SI}

A avaliação do sucesso dos SI nas empresas pode ser determinada por diversos fatores, e a escolha de uma medida que possa avaliar o sucesso dos mesmos está diretamente ligada ao desempenho da organização e desta forma muitas são as formas de avaliá-lo. De acordo com Maçada et al. (2005), na literatura não existe um consenso sobre qual medida atinge os objetivos de determinar o sucesso de um SI.

Desde a década de 1980, pesquisas que buscam avaliar o êxito de um sistema de informação têm sido realizadas, mas têm tratado diferentes aspectos desse tema, tornando difícil a comparação e acumulação dos resultados obtidos (DELONE; MCLEAN, 1992). Diante dessa percepção, os autores apresentam uma taxonomia, revisada em 2002, abrangente sobre o conceito de sucesso de um sistema de informação que tem sido explorada e ampliada em diversos estudos.

A taxonomia proposta inclui seis dimensões de sucesso do sistema de informação:

- Qualidade do sistema: envolve as características desejadas do sistema de informação, ou seja, preocupa-se com o sistema de processamento da informação;

- Qualidade da informação: enfatizam-se as saídas do sistema de informação, isto é, seus relatórios ou a qualidade do produto, que é a informação;

- Qualidade do serviço: preocupa-se em como as dimensões de qualidade afetam o uso e a satisfação do usuário;

- Intenção de uso: mensura a atitude ao invés de um comportamento do usuário em relação ao sistema em seu uso;

- Satisfação do usuário: preocupa-se com atitudes que independem da qualidade do sistema ou da informação;

- Benefícios Líquidos: envolve o impacto individual que se refere a uma indicação de que um sistema de informação tem produzido uma mudança na atividade do usuário ou alterado a percepção do tomador de decisão quanto à importância ou à utilidade de um sistema de informação; e também, o impacto organizacional, relaciona-se com a influência que o sistema de informação tem sobre a 
TOC e ABM: Uma Análise Comparativa à Luz da Literatura Especializada em Sistemas de

Carlos Roberto Kerber, Rodrigo Barreiros Leal, Ronaldo Francisco dos Santos, Anderson Chaves Da Silva, Carlos Alexandre Nascimento Wanderley, Samuel Cogan

organização.

Além disso, são apresentadas por Seddon (1997) quatro novas variáveis:

- Expectativa: é a projeção de quem toma a decisão, referente a custos e benefícios do uso futuro do sistema de informação;

- Consequências: podem ser societárias (acionistas), organizacionais e individuais dos resultados atribuídos ao uso do sistema de informação;

- Utilidade: refere-se ao aproveitamento percebido, representado por um indicador de percepção do nível em que o gestor acredita que o uso de um sistema de informação facilita o seu desempenho no trabalho; e

- Benefícios: são os proveitos líquidos percebidos para a sociedade e acionistas, assim como para os usuários.

\subsection{Teoria das Restrições (TOC)}

Conforme aduz Cogan (2007), o físico israelense Eliyahu Goldratt publicou em 1984 o livro chamado "A Meta" em que descreveu a teoria das restrições, desenvolvida a partir da década de 1970. Segundo Noreen et al. (1996), inicialmente a TOC foi construída por Goldratt que, por sua vez, elaborou seu próprio método de administração da produção. Nessa elaboração, utilizou o processo de raciocínio aplicado à física na resolução de problemas de logística de produção enquanto auxiliava um amigo na busca de melhorias no processo de gestão da empresa. Assim, Goldratt criou um sistema de contabilidade gerencial da Teoria das Restrições, identificado como Contabilidade de Ganhos, em que se mede o impacto de uma decisão no lucro líquido e no retorno sobre o investimento de uma empresa, sem incorrer em alocação de custos.

Segundo Gusmão (2004), a TOC adota princípios sistêmicos, por considerar que a melhoria do desempenho deve acontecer no nível sistêmico e não no nível de processos em particular. Assim, o caráter de ferramenta gerencial sistêmica que a TOC apresenta impõe que todos os aperfeiçoamentos devem ser realizados em relação à meta da organização, principalmente porque a busca do resultado global será sempre mais efetiva do que a busca de resultados parciais. 
TOC e ABM: Uma Análise Comparativa à Luz da Literatura Especializada em Sistemas de

A ideia principal em que se fundamenta a teoria é de que qualquer sistema, dentro de um contexto empresarial, tem sua produção limitada por, pelo menos, uma restrição que pode ser física (materiais, mercado, máquinas, fornecedores,) ou política (procedimentos, normas). Essa afirmativa assegura que nenhuma produção seja infinita. Nesse contexto, faz-se necessário uma clara definição do que seja uma restrição. De acordo com Noreen et al. (1996), representa qualquer coisa que impeça um sistema de alcançar seu objetivo. Ainda sobre o conceito de restrição, Guerreiro (1999) a define como tudo aquilo que limita um melhor desempenho de um sistema. Para Umble e Srikanth (1990), restrição é qualquer elemento que limita o sistema no cumprimento de ganhar dinheiro, limitando, portanto, seu desempenho.

A TOC estabelece princípios para a otimização da produção em um processo de melhoria contínua visando identificar a restrição, gerenciá-la e subordinar todos os recursos a esta restrição, formula um modelo de tomada de decisões e propõe formas de medição de desempenho (COGAN 2007).

\subsubsection{Medidas de Desempenho na Teoria das Restrições}

A TOC considera que o principal objetivo da empresa é sempre ganhar mais dinheiro e, em decorrência disto, Goldratt (2002) estabelece o uso de medidas financeiras para o acompanhamento do desenvolvimento da empresa em sua avaliação de desempenho.

Segundo Goldratt (2002), para julgar se a empresa está indo em direção à sua meta é necessário responder três perguntas: Quanto dinheiro é gerado pela nossa empresa? Quanto dinheiro é capturado pela nossa empresa? Quanto dinheiro deve-se gastar para operá-la?

Para responder a tais perguntas, o autor propõe uma mudança conceitual e sugere a adoção dos seguintes parâmetros operacionais:

- Ganho: É o preço de venda deduzido dos custos variáveis.

- Custos variáveis: São os gastos com materiais, comissões de venda, taxas alfandegárias, transportes, etc.

- Inventário: Todo o dinheiro investido na compra de coisas que se pretende vender ou que ajude na produção, como: estoques de matérias primas, produtos 
TOC e ABM: Uma Análise Comparativa à Luz da Literatura Especializada em Sistemas de

em processo e produtos acabados, máquinas e construções. Corresponde ao montante de recursos adquiridos e não consumidos.

- Despesa Operacional: Todo o dinheiro gasto para transformar inventário em ganho, ou todo o dinheiro gasto em algo que não pode ser guardado. Gastos no processo de transformação como mão de obra, energia elétrica e bens desgastados como depreciação de máquinas fazem parte das despesas operacionais.

Cogan (2007) resume ganho como o dinheiro que entra; inventário como o dinheiro atualmente no sistema, e despesa operacional como o dinheiro que tem que ser desembolsado para que o ganho aconteça.

Para avaliar o desempenho das empresas, a TOC recomenda o uso de medidas de alcance da meta através do uso dos parâmetros operacionais. As medidas de alcance da meta são:

- Lucro Líquido: Mede em termos absolutos quanto de dinheiro a empresa está gerando e é diferente do lucro líquido contábil.

Lucro Líquido = ganho - despesa operacional

- Retorno sobre Investimento: Dimensiona em termos relativos o esforço necessário para o alcance de determinado nível de lucro.

Retorno sobre Investimento = Lucro líquido / inventário

- Fluxo de Caixa: Não é considerada por Goldratt uma medida de desempenho, mas sim uma situação necessária para sobrevivência da empresa.

Princípios para a otimização da produção: de acordo com Guerreiro (1999), a Teoria das Restrições estabelece nove princípios básicos para a otimização da produção:

a) Balancear o fluxo e não a capacidade;

b) O nível de utilização de um recurso não gargalo não é determinado por seu próprio potencial e sim por outra restrição do sistema;

c) A utilização e a ativação de um recurso não são sinônimos;

d) Uma hora perdida no gargalo é uma hora perdida no sistema inteiro; 
TOC e ABM: Uma Análise Comparativa à Luz da Literatura Especializada em Sistemas de

Carlos Roberto Kerber, Rodrigo Barreiros Leal, Ronaldo Francisco dos Santos, Anderson Chaves Da Silva, Carlos Alexandre Nascimento Wanderley, Samuel Cogan

e) Uma hora economizada onde não é gargalo é apenas uma ilusão;

f) Os gargalos governam o ganho e o inventário;

g) Lote de transferência muitas vezes não deve ser igual ao lote de processamento;

h) Lote de processo deve ser variável e não fixo; e

i) Os programas devem ser estabelecidos considerando todas as restrições simultaneamente.

Otimizar a utilização da capacidade dos gargalos, segundo Guerreiro (1999), é um dos pontos básicos da TOC e é efetuada evitando o desperdício do tempo, ou seja, trabalhando durante todo o tempo disponível nos gargalos. Os gargalos não devem trabalhar para atender pedidos futuros em detrimento de pedidos atuais.

Os ensinamentos de Goldratt remetem para a definição de cinco passos que guiam o processo de melhoramento contínuo. São eles: identificar a (s) restrição (ões); decidir como explorar as restrições do sistema; subordinar qualquer outra coisa à decisão acima; elevar as restrições do sistema; e se nos passos anteriores uma restrição foi quebrada, volte ao passo 1 , mas não deixe que a inércia se torne uma restrição do sistema.

\subsubsection{Processo de Raciocínio}

De acordo com Noreen et al. (1996), a doutrina e a prática mostram que, após solucionados os problemas de ordem física na empresa, por meio dos conceitos aplicados da TOC, eles tendem a evoluir para problemas de ordem política. O processo de raciocínio, então, surge para resolver esses últimos. Em suma, o Processo de Raciocínio baseia-se em uma lógica de causa e efeito. O objetivo é descobrir o problema que está causando a restrição, ou seja, o problema verdadeiro. Nesse sentido, são três as perguntas que devem ser respondidas: $O$ que mudar? Para que mudar? Como fazer para mudar?

Cinco ferramentas estão disponíveis para responder a essas perguntas:

1) Árvore da Realidade Atual (ARA);

2) Diagrama de Resolução de Conflitos (DRC) ou Diagrama de Dispersão de 
TOC e ABM: Uma Análise Comparativa à Luz da Literatura Especializada em Sistemas de

Nuvem (DDN) ou simplesmente Nuvem;

3) Árvore da Realidade Futura (ARF);

4) Árvore de Pré-Requisitos (APR); e

5) Árvore de Transição (AT).

São vários os campos e situações em que o Processo de Raciocínio é usado. Diversos são casos de sucesso relatados, tais como diagnosticar problemas em cumprir os programas de produção e falhas nos projetos exclusivos de produtos e desenvolvimento de processo; tratar de problemas relativos a lucros baixos, sistemas de informação gerencial pobres, programas de aperfeiçoamento fracassados, até problemas com fornecedores; melhor decisão para construção de novas instalações (NOREEN et al., 1996).

\subsection{Sistema de Gerenciamento Baseado em Atividades - ABM}

O gerenciamento e controle das atividades empregando o Sistema de Custeio finalidade de estabelecer o preço de venda e a composição do mix de produtos e outras decisões. O ABM objetiva o gerenciamento do custo para o aperfeiçoamento e inovação do processo de produção ou da prestação do serviço, utilizando uma metodologia que se concentra na gestão das atividades como o caminho para a melhoria do valor recebido pelo cliente e dos lucros alcançados com o fornecimento desse valor. Além disso, inclui a análise das atividades e a medição do desempenho.

Para Cogan (1997), o ABC fornece as informações e o ABM as utiliza para as várias análises que objetivam o melhoramento contínuo da produção. O processo de implementação do $A B M$ tem como requisito que o $A B C$ já esteja implantado na empresa. O sistema ABM utiliza como principal fonte de informação a análise baseada em atividade, e tem como principal sistema de informação o sistema $A B C$. Este modelo de gerenciamento permite que a empresa atinja seus objetivos com menos recursos. Nesse sentido, Kaplan e Cooper (1998) apontam que o ABC levou naturalmente ao Gerenciamento Baseado na Atividade, que se configura como um conjunto de medidas interligadas, que só podem ser tomadas com base em informações provenientes de sistemas de custeio baseados na atividade.

Como visto, o sistema de gerenciamento baseado em atividades tem como 
finalidade auxiliar a empresa a atingir seus objetivos com o menor consumo de recursos, ou seja, obtendo os mesmos benefícios com um custo total. Esse objetivo somente será alcançado através de um conjunto de medidas interligadas, que somente podem ser desenvolvidas com informações provenientes do método de custeio $A B C$.

A gestão baseada em atividades pode ser utilizada para o estabelecimento de medidas de desempenho pelo balanceamento e melhoria da eficiência, da produtividade e da eficácia. O ABM poderá ser utilizado ainda como ferramenta de orçamento, estabelecendo a previsão orçamentária e planejando os recursos necessários, tomando-se por base as atividades (CHING, 2001).

Segundo Kaplan e Cooper (1998), a utilização do gerenciamento baseado em atividades poderá ser dividida em duas aplicações complementares: 0 ABM operacional e o ABM estratégico. O ABM operacional procura fazer as coisas como devem ser feitas, tem por objetivo aumentar a eficiência na realização das atividades, reduzir os custos e melhorar a utilização dos ativos, ao passo que o ABM estratégico almeja fazer as coisas que devem ser feitas tendo como objetivo a redução da quantidade de processos a fim de aumentar a lucratividade.

Segundo Ching (2001), enquanto o ABC é o processo técnico ou a mecânica para levantamento das atividades, rastreamento dos custos para as atividades e condução dessas atividades para produtos, clientes; o ABM é um processo que utiliza as informações geradas pelo ABC para gerenciar uma empresa ou um negócio, podendo-se afirmar que um banco de dados de atividades é a espinha dorsal, ou a base (fundamentos) do ABM. Ainda conforme o autor citado, a gestão baseada em atividades possui os seguintes princípios básicos:

- Os custos não ocorrem espontaneamente, eles são causados.

- Gerenciar as atividades e não os recursos e custos. Este princípio constitui-se no principal diferencial entre a gestão tradicional de custos e a baseada em atividades.

- Focalizar aquilo que origina a demanda por recursos, ou seja, os fatores geradores de custos, tomando-se ações para diminuir ou eliminar estes fatores geradores de custos. 
TOC e ABM: Uma Análise Comparativa à Luz da Literatura Especializada em Sistemas de

- Direcionar as atividades em função do cliente, questionando sempre se o cliente paga ou pagaria pela atividade que se está desenvolvendo.

- Otimizar os processos, reduzindo o número de atividades, tendo em vista o atendimento dos requisitos de agilidade, menor custo e maior qualidade.

- Procurar reduzir ou eliminar as atividades que não agregam valor, desnecessárias ou duplicadas.

- Melhorar continuamente as atividades.

- Executar as atividades consideradas corretas de forma contínua.

- O objetivo do gestor deve ser realizar a atividade correta o que é bem diferente de fazer a atividade do jeito certo.

Ainda segundo Ching (1997), o ABM apresenta algumas características sintetizadas, descrita abaixo:

- Custeio do Produto: análise da rentabilidade de produto/linha de produtos; racionalização da linha de produtos; e precificação.

- Custeio de cliente: análise de rentabilidade do cliente; estabelecimento de níveis de serviço de cliente.

- Gastos de capital: decisão de fazer ou comprar; e justificativa de investimento.

- Gerenciamento de processos: análise do valor do processo; melhoria do tempo do ciclo operacional; redução de desperdícios e duplicidades.

- Redução de custos: minimização ou eliminação de atividades que não agreguem valor; foco nos fatores geradores de custos.

- Medida de desempenho gerencial: balanceamento e racionalização da: Eficiência, Produtividade e Eficácia.

- Orçamento: planejamento de recursos necessários com base em atividades; e previsão orçamentária.

\section{ASPECTOS METODOLÓGICOS}

Quanto aos aspectos metodológicos, foi utilizada a pesquisa bibliográfica em razão da procura em explicar e discutir um assunto, tema ou problema com base em 
TOC e ABM: Uma Análise Comparativa à Luz da Literatura Especializada em Sistemas de

referências publicadas em livros, periódicos, sites, dentre outros. Dessa maneira, busca-se conhecer, analisar e explicar contribuições sobre determinado assunto, tema ou problema. Trata-se do levantamento de toda a bibliografia já publicada (MARTINS, 2007). Para Gil (2002), a principal vantagem desse tipo de pesquisa é permitir que o investigador cubra uma gama de fenômenos muito mais ampla do que aquela que poderia pesquisar diretamente.

$\mathrm{Na}$ revisão de literatura, são colocados os principais conceitos de sistemas de informação, abordando seus componentes de avaliação sob a dimensão da finalidade, sucesso e eficácia, bem como os conceitos relativos ao ABM e TOC. Logo após, é procedida uma análise desses SI discutidos, proporcionando comparações entre eles, de maneira a visualizar suas diferentes características e vislumbrar onde melhor se aplicam.

\section{ANÁLISE COMPARATIVA ENTRE TOC E ABM}

Ao se estudar a TOC e o ABM, verifica-se que estes SI possuem um enfoque gerencial, cuja finalidade é a de produzir informações necessárias para o processo de tomada de decisão na entidade. Denota-se que estes sistemas são pautados na análise da atividade, visando gerenciar os processos com intuito de que o consumo de recursos ocorra de forma eficaz, proporcionando, assim, um melhor desempenho.

Os sistemas ABM e TOC são, filosoficamente, similares. Ambos consideram a melhoria contínua através da remoção sistemática de desperdícios do sistema. Porém, na prática eles são bastante diferentes. Cada um dos sistemas inclui um número de técnicas que visam melhorar a competitividade e lucratividade da empresa sob certas condições. Ressalta-se que estes sistemas, por possuírem um foco gerencial, não visam atender as exigências legais e/ou fiscais.

Nesse cenário, o ABM se apresenta como um sistema predominantemente gerencial, cujo foco principal de análise é a atividade e enfoca a geração de informações relevantes para o processo decisório da empresa, buscando gerenciar os processos que consomem os recursos organizacionais para melhorar o desempenho da empresa como um todo. Já o modelo de gestão de custos proposto pela Teoria das 
Restrições (TOC) é pautado no gerenciamento eficaz da restrição, utilizando, entre outros critérios, o custeio variável. O enfoque principal da TOC é que toda empresa apresenta alguma restrição, que é definida como qualquer coisa que limite o melhor desempenho de uma empresa, como o elo mais fraco em uma corrente ou algo escasso dentro dos recursos da empresa.

Embora o ABM inclua um conceito de melhoria contínua, o seu principal elemento prático foca nas atividades contábeis, nas atividades de custos e nas atividades baseadas nos custos de produção, sendo assim considerado um sistema de gerenciamento contábil. Ao se utilizar do sistema $A B C$, que fornece precisamente os custos de produção de acordo com as atividades, através do uso de direcionadores de custos, o ABM reforça a ideia de ser um sistema de informação contábil.

A TOC estabelece o foco na melhoria dos processos na empresa, reduzindo os desperdícios e balanceando o fluxo de trabalho, gerando informações contábeis de caráter gerencial em relação à capacidade produtiva, necessidade e tamanho dos estoques, informações estas oriundas do conhecimento das restrições ou gargalos produtivos.

O enfoque do ABM é o gerenciamento das atividades internas e da cadeia de valor (fornecedores, canais de distribuição e clientes) que é considerado, respectivamente, no $A B M$ operacional e no ABM estratégico (KAPLAN; COOPER, 1998). Nesse contexto, ele contempla tratamento da atividade através do centro de resultado, possui como objeto de acumulação de custos as atividades, produtos/serviços, fornecedores, clientes e canais de produção, como medidas de desempenho possui múltiplos indicadores que contemplam aspectos financeiros e não financeiros, buscando um equilíbrio entre eles.

A TOC visa identificar as restrições ou gargalos existentes no sistema produtivo da empresa, tendo como objeto de trabalho as atividades e processos executados, afetando toda a cadeia de valor. É uma nova filosofia de pensamento gerencial e sua premissa básica é gerenciar a partir das limitações ou restrições existentes no sistema, focalizando como objetivo econômico máximo (meta da empresa) o "ganhar dinheiro".

Por ser considerado um sistema de informação contábil, o ABM contempla 
TOC e ABM: Uma Análise Comparativa à Luz da Literatura Especializada em Sistemas de

diversos aspectos contábeis como: avaliação de ativo (recomenda o uso do custo corrente); depreciação, preço de transferência, lucro (utiliza-se do Valor Econômico Agregado - EVA), reconhecimento da receita, ocorrendo quando a empresa executa uma atividade, etc. Ademais, o Gerenciamento Baseado nas Atividades é um modelo de gestão que tem como uma das suas premissas a melhoria contínua, formação e agregação de valor para o cliente (CHING,1997), e que utiliza, normalmente, as informações fornecidas pelo ABC (Activity Based Costing) para a tomada de decisão.

A TOC também é considerada um sistema de informação contábil. Estabelece uma nova base conceitual para elementos contábeis, tais como o Ganho, o Lucro, a Despesa Operacional e, ainda apresenta um modelo de mensuração por meio da utilização de medidas como o lucro, Retorno sobre o Investimento (ROI), além de considerar uma condição necessária através da qual pode se garantir a sobrevivência da empresa e que é chamado fluxo de caixa.

\subsection{Dimensão da Eficácia}

Os sistemas de informações ABM e TOC ao serem analisados sob a ótica da dimensão da eficácia apresentam uma capacidade de produzir informações em tempo hábil. Porém, ambos os sistemas não podem ser considerados simples e rápidos, haja vista a complexidade de seus conceitos e teorias adjacentes que regulam a sua operacionalidade. Esta complexidade afeta substancialmente o fluxo dos processos no quesito velocidade, mas cria uma racionalidade nos mesmos, promovendo a integração das diferentes unidades organizacionais pelo delineamento das atividades envolvidas.

\subsection{Dimensão da Finalidade}

Como resultado da análise da TOC e do ABM, sob a ótica da dimensão da finalidade, ambos os sistemas propiciam a redução dos custos das operações gerenciais, diminuindo o uso da mão de obra administrativa, possibilitando uma descentralização da autoridade no processo decisório que é aperfeiçoado com a melhora ao acesso das informações em atendimento as necessidades gerenciais. Ainda, estes sistemas tendem a apresentar uma melhora na estrutura organizacional e hierárquica, diretamente relacionadas ao conhecimento das atividades e ao 
TOC e ABM: Uma Análise Comparativa à Luz da Literatura Especializada em Sistemas de

funcionamento dos sistemas e suas premissas com elevado ganho de motivação de pessoal, e estreitamento das relações da entidade com os seus diversos colaboradores internos e externos.

Denota-se que no componente "projeção de resultados", o ABM apresenta soluções seguras para o planejamento em longo prazo, tendo melhor desempenho no nível de gestão estratégico, enquanto que a TOC demonstra melhor proeminência em curto prazo, sobressaindo-se no nível de gestão tático e operacional. Em face destas características que diferenciam o desempenho nos horizontes de planejamento e nos níveis de gestão, a TOC apresenta uma melhor capacidade de resposta aos acontecimentos não-previstos.

No componente "ganhar produtividade", observa-se uma diferenciação, em que no $\mathrm{ABM}$ há a premissa que busca a maximização do resultado pela utilização plena da capacidade produtiva, enquanto que na TOC ocorre a otimização da produção com a identificação e eliminação dos gargalos.

\subsection{Dimensão de Sucesso}

A avaliação do sucesso dos SI, ABM e TOC, demonstra que a qualidade do sistema de informação está pautada na qualidade da informação que, em ambos, é acessível, caracterizada de forma flexível e confiável. A implantação destes SI impõe a sua utilização continua com melhoria do processo de tomada de decisão e consequente satisfação pela qualidade e tempestividade das informações produzidas.

Os impactos são medidos com o ganho da melhoria da qualidade das informações e do desempenho operacional, em que os usuários passam a participar dos processos e a reconhecer a satisfação de suas necessidades, cujas expectativas são atendidas com consequências mensuráveis no resultado das atividades envolvidas. Soma-se a isso o fato de que a utilidade destes SI são percebidas por meio de seus indicadores de desempenho, demonstrando os benefícios alcançados pelo uso e aplicação do sistema de informação. 
TOC e ABM: Uma Análise Comparativa à Luz da Literatura Especializada em Sistemas de

Informações Gerenciais

Carlos Roberto Kerber, Rodrigo Barreiros Leal, Ronaldo Francisco dos Santos, Anderson Chaves Da Silva, Carlos Alexandre Nascimento Wanderley, Samuel Cogan

\section{CONSIDERAÇÕES FINAIS}

Fiel ao propósito do trabalho, dois sistemas de informações contábeis foram abordados e foram avaliados quanto às três dimensões (finalidade, eficácia e sucesso) para verificar a abrangência e alcance dos SI propriamente ditos. Assim, com esta análise, foi possível concluir que o ABM e a TOC, mesmo com algumas restrições ou 0 não atendimento de algum requisito, são considerados SIC que atendem a finalidade que se propõem de forma eficaz e com o sucesso esperado.

Observa-se que a visão sistêmica apresentada contribui para a compreensão do funcionamento das técnicas e ferramentas inerentes a cada SIC. Nota-se que é admissível identificar as possíveis interações entre o ABM e a TOC nos diversos processos decisórios, na identificação das restrições e "gargalos", dos problemas potenciais e efetivos, e as oportunidades de melhorias contínuas e maximização dos resultados com uma produção adequada à capacidade implantada, conforme observado em suas principais características. A TOC tem como base a geração da riqueza através da eliminação de restrições, tendo como foco principal a otimização da produção e a eliminação de "gargalos". Além disso, suas medidas de desempenho são financeiras e devem expressar a meta. Com relação ao ABM, sua base é o sistema de custeio por atividade. Proporciona também uma ampla reengenharia dos processos de negócios, apresentando melhores medições de desempenho e por possuir um horizonte de longo prazo, oferece informações mais precisas para propostas de investimentos.

No que se refere à gestão de custos, a TOC é baseada em custeio variável sob a ótica dos novos conceitos introduzidos. Possui também foco inteiramente voltado no processo de manufatura e produção, não atribuindo importância aos aspectos voltados à gestão financeira e é um modelo orientado para curto prazo, sem conotações estratégicas. Já o $A B M$ é um sistema totalmente dependente do custeio $A B C$, possuindo o foco nos custos e processos, e, consequentemente, incorre em dificuldades para identificação da responsabilidade dos custos fixos.

Quanto à aplicabilidade dos SIC e um sistema ERP, ambos os sistemas podem ser utilizados com fins gerenciais. A TOC é aplicada em sistemas de nível operacional 
TOC e ABM: Uma Análise Comparativa à Luz da Literatura Especializada em Sistemas de

e tático para controle da produção e manufatura integrado ao Sistema OPT. Doutra sorte, o ABM é aplicado em sistemas de nível estratégico. Denota-se, contudo, que os responsáveis pela geração das informações contábeis precisaram de profundos conhecimentos nas técnicas aplicadas nos diversos sistemas para corretamente assessorar os usuários destas informações, elementos estes que também precisaram ter o conhecimento para utilizar as informações proporcionadas nos processos de tomada de decisão.

Assim, conclui-se que os SIC devem possuir a capacidade de oferecer uma diversidade de informações contábeis e financeiras que permitam atender as diferentes necessidades dos diversos níveis de gestão nos pertinentes processos de tomada de decisões. O atendimento deste requisito propiciará a empresa, um incremento qualitativo no seu controle interno com uma maior integração entre os diversos setores e usuários. Como visto, o ABM e a TOC atendem níveis administrativos diversos e, consequentemente, podem ser usados em conjunto e em complemento ao suporte necessário à tomada de decisão de acordo com o horizonte vislumbrado.

Não se pretendeu, com este estudo, esgotar as diversas maneiras de se avaliarem os sistemas de informação, ou evidenciar qual sistema é melhor ou pior, mas sim, apresentar uma forma de avaliação, seguindo as sugestões propostas por pesquisadores da área. De qualquer maneira, fica claro que a utilização de um sistema em detrimento de outro ou a combinação de ambos é uma decisão que deverá considerar diversas variáveis intrínsecas ao interessado e usuário da informação contábil gerada, assim como do horizonte (curto prazo e longo prazo) e do nível de gestão (operacional, tático ou estratégico) a ser contemplado com o uso e benefícios proporcionados. Por fim, sugere-se a continuidade desta pesquisa com um estudo empírico analisando as possíveis limitações do uso dos sistemas em conjunto.

\section{REFERÊNCIAS}

ALTER, S. Information Systems, 2nd ed., The Benjamin/Cummings Publishing Company, Inc., Menlo Park, CA. pp. 23-25, 1996.

ANTHONY, R. N. Contabilidade Gerencial. São Paulo: Atlas, 1972, 488 p. 
TOC e ABM: Uma Análise Comparativa à Luz da Literatura Especializada em Sistemas de

Informações Gerenciais

Carlos Roberto Kerber, Rodrigo Barreiros Leal, Ronaldo Francisco dos Santos, Anderson Chaves Da Silva, Carlos Alexandre Nascimento Wanderley, Samuel Cogan

CHING, H. Y. Manual de custos de instituições de saúde: sistemas tradicionais de custos e sistema de custeio baseado em atividades (ABC). São Paulo: Atlas, 2001, 256 p.

CHING, H. Y. Gestão baseada em Custeio por Atividades. São Paulo: Atlas, 1997, 184 p. COGAN, S. Modelos ABC/ABM. Rio de Janeiro: Qualitymark, 1997, $188 \mathrm{p.}$

. Contabilidade Gerencial: Uma Abordagem da Teoria das Restrições. São Paulo: Saraiva, 2007, 294 p.

DELONE, W. H.; MCLEAN, E. R. Information systems success - the quest for the dependent variable. Information Systems Research. 3(1), 1992, p. 60-95.

Information systems success revisited. In: Proceedings of Hawaii International Conference on System Sciences, Proceedings 35th, Big Island, Hawaii, 2002.

DIAS, G. M. A.; MOURA, I. J. L.; SILVA, M. V. P. e CARVALHO, J. D. S. G. 2003. O uso dos sistemas de informações contábeis como apoio nas decisões gerenciais. In: CONGRESSO SERGIPANO DE CONTABILIDADE II, Anais..., Aracajú, 2003.

GIL, A. C. Como elaborar projetos de pesquisa. 4. ed. São Paulo: Atlas, 2002, $184 \mathrm{p}$.

GOLDRATT, E. M. e COX, J. A Meta: um processo de melhoria contínua. São Paulo: Nobel, 2002, 366 p.

GUERREIRO, R. A Meta da Empresa: Seu Alcance sem Mistérios. 2. ed. São Paulo: Atlas, 1999, $133 \mathrm{p}$.

GUSMÃO, S. L. L. de. Proposição de Um Esquema Integrando a Teoria das Restrições e a Teoria dos Custos de Transação para Identificação e Análise de Restrições em Cadeias de Suprimentos: estudo de casos na cadeia de vinhos finos do Rio Grande do Sul. Porto Alegre, RS. Tese de doutoramento. Universidade Federal do Rio Grande do Sul - UFRGS, 2004, 222 p.

HORNGREN, C.T.; DATAR, S. M.; FOSTER, G. Contabilidade de custos. 2 vol, 11 ed., São Paulo: Pearson Prentice Hall, 2004, 846 p.

KAPLAN, R. S.; COOPER, R. Custo e desempenho: administre seus custos para ser mais competitivo. São Paulo: Futura, 1998, 376 p.

LAUDON, K. C. e LAUDON, J. P. Sistemas de informação: com internet. 4. ed. Rio de Janeiro: LTC, 1999, 387 p. 
TOC e ABM: Uma Análise Comparativa à Luz da Literatura Especializada em Sistemas de Informações Gerenciais Carlos Roberto Kerber, Rodrigo Barreiros Leal, Ronaldo Francisco dos Santos, Anderson Chaves Da Silva, Carlos Alexandre Nascimento Wanderley, Samuel Cogan

MAÇADA, A. C. G.; BECKER, J. L. e LUNARDI, G. L. Efetividade de Conversão dos Investimentos em TI na Eficiência dos Bancos Brasileiros. Revista de Administração Contemporânea, 9(1), 2005, p. 9-33.

MARTINS, G. de A.; THEÓPHILO, C. R. Metodologia de Investigação Científica Para Ciências Sociais Aplicadas. São Paulo: Atlas, 2007, 250 p.

MEIRELLES, F. S. Informática: novas aplicações com microcomputadores. São Paulo: Makron Books, ed. I, 1994, 615 p.

NOREEN, E. W.; SMITH, D; MACKEY, J. T. A Teoria das Restrições e Suas Implicações na Contabilidade Gerencial. São Paulo: Educator, 1996, 224 p.

O'BRIEN, J. A. Sistemas de informação e as decisões gerenciais na era da Internet. 2. ed. São Paulo: Saraiva, 2004, 526 p.

OLIVEIRA, D. de P. R. Sistemas de informações gerenciais. 6. ed. São Paulo: Atlas, 1999, $268 \mathrm{p}$.

PADOVEZE, C. L. Contabilidade Gerencial: um enfoque em sistema de informação contábil. São Paulo: Atlas, 2007, 632 p.

PEREIRA, G. R. Aplicação da Gestão Baseada em Atividades à Distribuição Urbana de Bebidas. Rio de Janeiro, RJ. Tese de doutoramento. Universidade Federal do Rio de Janeiro - UFRJ, 2007, 230 p.

POLLONI, E. G. F. 2000. Administrando sistemas de informação. São Paulo, Futura, $272 \mathrm{p}$.

RIELLA, C. G.; KREISIQ, D.; KAMMLER, E. L.; FRIGERI, J. A. A evolução dos sistemas e a nova era sistêmica. In: $X$ Congresso Brasileiro de Custos, Anais... Guarapari, 2003.

ROCHA. A. O Essencialdos Sistemas de Informação. 2001. Disponível em: <http://www.ufp.pt/staf/amrocha/publica.html>. Acesso em: 20 jan. 2010.

SAKURAI, M. Gerenciamento integrado de custos. São Paulo: Atlas. 1997, 280 p.

SEDDON, P. B. A respecification and extension of the DeLone and McLean model of is success. Information Systems Research. 8(3): 1997, 240253.

STRASSBURG, U.; FIORI, R.; PARIZOTO, K. G.; BAZZOTTI, C.; FONSECA, E. F. de M. A importância do sistema de informação contábil como fonte de informações para tomada de decisões. In: VI Seminário do Centro de Ciências Sociais Aplicadas de Cascavel, Anais... Cascavel, 2007. 
TOC e ABM: Uma Análise Comparativa à Luz da Literatura Especializada em Sistemas de Informações Gerenciais Carlos Roberto Kerber, Rodrigo Barreiros Leal, Ronaldo Francisco dos Santos, Anderson Chaves Da Silva, Carlos Alexandre Nascimento Wanderley, Samuel Cogan

Data de Submissão: $30 / 08 / 2010$

Data de Aceite: 29/07/2011 